\title{
Research on Promotion System of Nationwide Fitness Martial Arts
}

\author{
Linji An \\ Physical Education Institute, Baoshan University, Baoshan , 678000, China
}

Keywords: National wide fitness, Traditional martial arts, Martial arts promotion.

\begin{abstract}
In the 21st century, as the role of knowledge in society play an increasingly significant, humanity gradually into a knowledge society, although greatly promoted the social progress and development, but it also caused workers Yongnaoguodu, lack of exercise, resulting in adverse effects on public health. In the martial arts fitness as a certain social resistance, it can be widely recognized by the community sports should take certain measures to promote their health fitness function into full play, enhance the overall quality of the people to make a contribution.
\end{abstract}

\section{Introduction}

Fitness martial arts promotion system specifically is through martial arts fitness promotion basic structure and process of systematic analysis, and thus to promote the implementation of martial science and comprehensive description, and appropriate analysis in the promotion process in all aspects and elements of relations among variables. Fitness martial arts promotion system can research fitness and martial arts to promote a clear delineation of the scope, and then start from several related elements of the implementation of comprehensive analysis and research for the practical operation of martial arts promotion provide appropriate support and learn from, to deepen our fitness work force to carry out the appropriate contribution.

\section{The role of traditional Chinese martial arts in fitness work}

\section{The role of traditional martial arts theoretical basis}

Traditional Chinese martial arts in the development process of Chinese traditional culture as a theoretical basis for the classic, is a kind of offensive and defensive skills as the main content and inherited the Chinese people's self-defense and fitness technique. Its content is more abundant, and formed a diversified forms in the development process, the style is also influenced by traditional Chinese culture is unique, line efficiency significantly, for young and old to the younger exercises to strengthen the people's physique produce the corresponding positive influence. A long time to martial arts training, not only physical fitness, but also will strengthen the quality of people, prolong life expectancy, play an extremely important role in the current social fitness work.

\section{Traditional martial arts has a variety of application advantages}

Rich in content and diverse forms of traditional martial arts has, prompting the exerciser capable of binding to their own needs to get wider choices. In traditional martial arts used in the process of fitness work, whether it is currently in school students or younger elderly in society, no matter what profession, are able to explore their needs in accordance with the appropriate exercise of their own martial arts project [1]. Meanwhile, the martial arts exercise program more flexible, no movement and preparation of special sports clothing and venues, can be carried out anytime, anywhere. Especially for the lack of grass-roots enterprises, construction of sports funding situation, actively introduce martial arts fitness, with minimal investment for a larger output, play a better workout 
results. Therefore, in the current social context, with the actual situation of each region and actively carry out martial arts fitness work has certain advantages.

\section{Fitness martial arts promotion system}

Fitness martial arts promotion system contains many elements; the following article will be a specific analysis.

\section{Promoters in Fitness martial arts promotion system}

In martial arts fitness system extension staff consists mainly of government promoters widely deployed and private voluntary martial arts promoter. The official promotion of the general by the regional government faces fitness martial arts promotion activities and support organizations, and private promoters usually refer to widely spread in the community, for various reasons, self-organized martial arts promotion activities. For specific promotion, the promotion of the content and manner of official and private promoters may be selected by the presence of the same or different, such as official more emphasis on competitive martial arts promotion is an important part of the promotion of civil society.

\section{Fitness martial arts promotion system mainly to promote content}

Mainly to promote comprehensive health promotion martial arts systems involved typically include technical and cultural aspects, in accordance with the relevant classification specific division, with technology as the standard martial arts fitness promotion activities can be divided into two components of technical and non-technical technology promotion is to promote the martial arts skills, promote the general content rather than technology is relatively wide, such as wushu, martial arts aesthetics, martial fitness literature, martial Arts theory and related martial arts film promotion, these non-technical content belonging to cultural context and therefore it can be referred to the promotion of culture, so that martial arts promote fitness main contents include technical and cultural aspects.

\section{Implementation objects of fitness martial arts promotion system}

The main target is to promote fitness martial arts learners to promote their specific educational process can play a relevant impact on the promotion of the object. Fitness martial arts martial arts to promote the educational value of learners mainly in the following aspects: First, in terms of Takenori training, our fitness Takenori martial arts promotion will run through the promotion of all aspects of the work, whether it is for youth groups or adults want their learning through martial arts, it is possible to develop a ritual first, respect for the quality of the behavior of others [2]. Secondly, in the specific technical learning process, but also to the quality of the learner's exercise will cultivate confidence in learners, exercise and play a role in strengthening their physical and mental development. The presence of these two educational function fully reflects the educational fitness martial arts promotion, and worthy of further application and dissemination of contemporary society in strengthening national quality work.

\section{Promotion methods in fitness martial arts promotion system}

Way to promote fitness martial arts promotion system of choice generally comprises interpersonal promotion, promotion among organizations, networks and online promotion of several mass promotion, which mainly refers to the promotion of mutual relationships, and inheritance among people between master and apprentice communication and mutual instruction; the promotion of major organizations in the community or in the form of martial Arts Association to promote fitness martial arts community, in general, to promote the organization of a strong group can be in the form of groups of influence is relatively Great, it is a more popular way of promotion; specifically, to promote the public through books and newspapers to promote film and television works, promotion of fitness martial arts to enhance the effectiveness of the promotion; the network is to promote the use of computer network implementation of online promotion, so that more people recognize the value of martial arts fitness activities.

\section{Promotion of fitness martial arts promotion system}

In the fitness martial arts promotion system is generally used to promote the level of promotion level judge and divide. Level promotion mainly from the martial arts promote fitness range of content 
choices and to promote acceptance of martial arts training target content to specific division, including shallow, middle and deep three kinds of promotion. For example, in the beginning of this century, compose and organize the community to practice martial arts fitness aerobics should be divided into shallow promotion category [3]. In a promotion, since the martial arts aerobics fitness relates only to a part of martial arts training content, even if the masses of its high-intensity training, cannot truly comprehend the essence of martial arts fitness, so you can just say that this level of promotion in order to deepen the understanding of martial arts fitness for the masses, and not produce profound influence on the masses.

\section{The features of fitness martial arts promotion system}

In the study of the function of fitness martial arts promotion system is analyzed, it is possible to grasp the deeper comprehensive fitness martial arts promotion system works. From the specific method presented can be seen, fitness martial arts promotion has the function can generally be divided into two types of explicit and implicit; and released from the generated function-related effects were analyzed, it should be divided into both positive and negative functions. Specifically, to promote fitness martial arts promotion of good development, thereby promoting fitness martial arts in our country from generation to generation, combined with the needs of the times and people's physical needs to be strengthened in the tradition of innovation process, derived more suitable for the new school, to ensure that the role of martial arts in promoting fitness to enhance the overall national quality aspects can be fully realized.

\section{Fitness martial arts promotion system to promote the principles of the work carried out}

Combined with the actual needs of current fitness martial arts promotion, and in particular the promotion of the development process should adhere to the following principles:

1). The promotion of the principle of good faith. Promotion is to ensure the integrity of the promotion of the success of the key points, but also enhance the effectiveness of the promotion of the main contributing factor. Neither for the promotion of official or private promotion, good faith has a certain importance, as a department official extension workers extension work, in the organization of athletic competition should adhere to the principle of good faith, to ensure fair and equitable competition, and establish in the community good image; and civil martial arts promoters should also comply with the principle of good faith, to provide the appropriate safeguards for the sustained and healthy development of their own martial arts promotion activities.

2). For promotion. And selectively carry out promotional work for the current fitness martial arts promotion in the purpose of promotion and other aspects of population differences exist. For such children should select the appropriate promotion simplify routine, appropriate for women can be promoted to the Magnolia project, Tai Chi and other items for the older age groups. Only the actual needs of the masses to carry out the promotion ensure the promotion of the work to obtain the corresponding success.

3). Hierarchical promotion. In the martial arts promote fitness layering system to promote the principles should belong to the research areas of epistemology, has some guiding significance for the promotion of specific social reality. As the actual work will be promoted fitness martial arts can be divided into shallow promotion, taking into account students' poor understanding of martial arts, the ability to accept the status quo to a general weak ordinary institutions. Chinese martial arts in the dissemination and development of China is divided into three phases junior high, it is based on the theory of hierarchical promotion, in order to meet the learning needs of different groups, to promote sustained and healthy development of the work produced by a corresponding positive impact. .

\section{Strategy of popularizing martial arts and promoting fitness}

\section{The martial arts promotion and school physical education curriculum combined}

An important purpose of organized school sports teaching is that students learn a movement of the two corresponding skills, and develop the habit of lifelong sports consciousness and culture through reasonable teaching practice, encourage students to get better development in the future. So will adhere to the basis of the traditional martial arts Teaching of Physical Education teaching into 
practice, it is possible to promote the advantages of martial arts teaching sports to get better results, enhance the integrity of the National Quality produce a corresponding positive impact [4]. In PE teaching in primary, teachers can promote students to appropriate some of the traditional martial art form has a strong interest, so that students from basic education stage to accept the cultural influence of martial arts education and to enhance students' physical fitness, sports teaching quality lifting foundation. In the intermediate and advanced education should be combined with the development stage features a higher level of penetration of student martial arts education, to promote National Fitness Program With the development of martial arts education to give the corresponding implementing to senior colleges martial arts fitness activities driven the whole community to actively participate in martial arts fitness to produce the corresponding catalytic influence on increasing the quality of our nationals. Universities Present levels of positive response to the call of the national fitness has basically opened a martial arts course, hire a professional martial arts teachers, students implement fitness martial arts education, hoping to further strong student body, students will form a good quality but also further promote the fitness of martial arts promotion efforts.

Martial arts community attaches great importance to education, to promote the development of martial arts education towards entities.

Martial Arts promotional support needs of our community's strength in implementing the national fitness thought process. In concrete work, the needs of all levels of administration can be combined with martial arts promotion configuration corresponding social martial arts instructor, according to the site to carry out the reasonable needs of each community and the masses of teaching martial arts, such as tai chi training organization appropriate to set up martial arts fans house, etc., to protect people in the community can participate in a wide range of community education in martial arts, martial arts promote fitness under the background of education and public entities gradually toward development, really play the advantages of education in martial arts to enhance national quality aspects [5].

Improving information and education, and expand the impact of martial arts education In order to promote the national fitness martial arts education to better integrate into society, to play a greater development effectiveness, resulting in a corresponding positive impact on the promotion of national quality, provide more quality services for the community. At this stage, the authorities can collect a number of high educational level of martial arts experts, publicity and promotion fitness martial arts education in-depth research, and guidance systems theory to construct a reasonable guide mode, which enables it to promote its folk from separated, independent stable development, and promote good conduct fitness work.

\section{Conclusions}

In summary, the martial arts promote fitness in the current background to enhance the integrity of the National Quality produce a corresponding positive impact, so the community should pay greater attention to the promotion of martial arts fitness, with the relative scientific promotion system proposed the promotion of appropriate measures to promote public awareness of the importance of learning martial arts, to enhance interest in learning martial arts, martial arts promote fitness promotion to get healthy development, protection of martial arts promotion of social function can be fully exploited.

\section{References}

[1] Ke Yingjun, Song Liang. Study on role of martial art in fitness and promotion strategy, Cifeng College Journal(natural science edition), 2013(9):158-160.

[2] Fang Bin. Advantages and Strategies of Henan Province Wushu Rank promotion under National Fitness, Sports, 2014(24):149-150. 
[3] Wang Mingjun, Yan Yan, Du Shougao. Discussion on establishment of popular fitness promotion model from taekwondo hot, Sports scientific literature,2013,21(8):93-95.

[4] Liu Yundong, Zhao Yu, Li Tingting. On the Role of martial art in National Fitness and extension development, Fitness Aerobics, 2015,12(3):29-30,49.

[5] Wang Qinglong, Liu Wenbao. Role and Strategy of Promoting martial art in Cangzhou City National Fitness Program, Huaibei vocational and technical college Journal,2011,10(4):141-142. 